\title{
Impacto del consenso de grupo y el apoyo social en el significado del estímulo: Mediación de la reestructuración cognitiva en la conformidad
}

\section{Vernon L. Allen}

Universidad de Wisconsin-Madison

\section{David A. Wilder}

\section{Universidad estatal de Rutgers}

FUENTE: Journal of Personality and Social Psychology, 1980, 39, 1116-1124

Se llevaron a cabo tres experimentos para probar la explicación de la conformidad basada en la reestructuración cognitiva. La bipótesis era que las personas reinterpretan el significado del objeto estimular cuando se enfrentan a respuestas impopulares de un grupo unánime, y que éste cambio en el significado lleva a un cambio de la respuesta bacia la postura del grupo. En el primer experimento, se presentaron varios items de opinión, y los sujetos observaban respuestas impopulares, supuestamente emitidas antes por un grupo unánime o por un grupo que tenia un individuo disconforme (apoyo social), o bien no observaban ninguna respuesta en absoluto (control). Los sujetos simplemente daban su interpretación del significado de una palabra o frase clave de cada manifestación de opinión -no daban sus propias opiniones. Los re- sultados mostraron que los sujetos del grupo unánime daban más significados infrecuentes a los estímulos que los sujetos de las condiciones de apoyo social y de control. Asi pues, el contexto social provocaba un cambio en el significado del estimulo. El experimento 2 excluía la posibilidad de que la desviación en el significado fuera debida a la adbesión por parte de los sujetos a la interpretación que suponian era mantenida por la mayoria. En un tercer experimento, se exponía a los sujetos a puntuaciones que representaban los significados producidos por las condiciones de grupo unánime y de control del experimento 1. Los resultados mostraron que después de observar los significados producidos por consenso para estos items, los sujetos desviaban sus propias opiniones bacia la postura mantenida por el grupo unánime 
del experimento 1. Así pues, un cambio en el significado de los estímulos era responsable de un cambio de la opinión.

Desde el clásico estudio sobre conformidad dirigido por Asch (1951) se han dado varias explicaciones teóricas del descubrimiento bien documentado de que la conducta de un individuo se ve influida por un grupo unánime. Las discusiones anteriores sobre el conformismo se han centrado sobre todo en el papel de factores motivacionales y sociales. Este estudio ofrece un análisis teórico que difiere de las explicaciones tradicionales del conformismo.

Lo que proponemos es que el consenso de grupo produce conformismo de una manera directa, mediante la modificación del significado del estímulo mismo. Este análisis supone que el impacto del grupo en la conducta del individuo se expresa en una secuencia de dos pasos. En primer lugar, el grupo unánime modifica la interpretación que hace el sujeto del estímulo que se está juzgando. En segundo lugar, la reinterpretación del estímulo hace que la postura a la que se adhiere el grupo parezca más aceptable, llevando por esa razón al sujeto a pasarse a la postura del grupo.

Según este análisis, el impacto del grupo sobre la conducta del individuo opera de una forma más indirecta y sutil de lo que reconocen la mayoría de las demás explicaciones del conformismo.

Expuesto en términos más generales, lo que estamos afirmando es que el significado atribuido a un estímulo se ve influido por el contexto en el que aparece. Por supuesto que esta noción no es de ninguna manera una concepción nueva ni sorprendente; se reconoce como una de las proposiciones básicas de la psicología de la Gestalt y ha sido aplicada con éxito a problemas de psicología social (Asch, 1940, 1948; Newtson y Czerlinsky, 1974). Este principio fue empleado por Asch (1948) en su reconstrucción de la conceptualización tradicional de los fenómenos de influencia social. Asch insistía en que el cambio de actitud no representa un cambio en la evaluación del objeto, sino un cambio en el objeto que está siendo evaluado. En otras palabras, la persona reestructura cognitivamente el objeto de evaluación y el significado del problema experimenta una transformación en función del contexto que tiene lugar.

Las pruebas experimentales de la tesis de Asch han sido escasas y en gran parte anecdóticas. Uno de los primeros estudios exploró el fenómeno de la sugestión por prestigio (Asch, 1948): el deseo de los sujetos de estar de acuerdo con una afirmación se mostró que variaba en función de la autoría atribuida. Por ejemplo, los sujetos se inclinaban más a estar de acuerdo con una cita que recomendaba una revolución cada veinte años cuando se atribuía de Thomas Jefferson (el autor real) que cuando se creía que el autor era Lenin. El conocimiento de la autoría alteraba el significado dado al objeto de evaluación (la cita). Asch afirmaba que el grado de acuerdo con la declaración podría estar vinculado a la diferencia en la definición de los sujetos o el significado relacionados con el objeto (p. ej., el significado de una palabra clave como «revolución»).

Según la teoría y los datos pertinentes discutidos por Asch $(1940,1948)$, la manera en que otra persona (o un grupo) juzga un asunto puede alterar la interpretación que hace el individuo de este asunto. Es decir, el contexto social sirve para transformar el significado del estímulo. En la situación de conformidad, un aspecto importante del contexto social es la estructura del grupo, sobre todo la unanimidad de las respuestas de los miembros del grupo. En este estudio, nuestra hipótesis es que un grupo unánime cambiará la interpretación que da el sujeto a un estímulo de opinión. Si la comparamos con el significado del mismo estímulo en presencia de un grupo no unánime o cuando falta información sobre las respuestas de otras personas.

El análisis de la reestructuración cognitiva puede arrojar luz sobre uno de los descubrimientos estables en el área del conformismo o la reducción del confor- 
mismo producida por la presencia de un apoyo social (un aliado). Cuando un miembro del grupo no está de acuerdo con el grupo equivocado - sino que está de acuerdo con la postura privada del sujeto-, el conformismo se reduce de forma notable e inmediata (Allen, 1975; Asch, 1951). Nuestra hipótesis es que el apoyo social reduce la conformidad de forma eficaz porque evita la desviación del significado del estímulo, que normalmente tiene lugar cuando un individuo se enfrenta a las respuestas unánimes de un grupo. En el primer experimento, los sujetos observaron bien respuestas de un grupo unánime (presión unánime), o bien ninguna retroalimentación referente a las respuestas de otras personas (control). Luego los sujetos daban su interpretación del significado de una palabra o frase clave para cada una de las diversas manifestaciones de opinión. Es importante recalcar que a los sujetos no se les pedía que dijesen cuáles eran sus evaluaciones (acuerdo o desacuerdo) sobre los enunciados de opinión. Por lo tanto, cualquier diferencia entre las condiciones experimentales en cuanto al significado de los enunciados puede atribuirse al contexto social en que aparecían las afirmaciones: grupo unánime, grupo con un disconforme, o ausencia total de información sobre la opinión (retroalimentación) al grupo.

\section{EXPERIMENTO I}

\section{METODO}

\section{Sujetos}

Los sujetos eran 93 estudiantes no graduados (44 vatones y 49 mujeres) de una gran universidad estatal. Todos los sujetos estaban matriculados en clases introductorias de psicología y recibían un crédito extra por participar en la investigación. Los datos de 4 sujetos ( 3 varones y 1 mujer) fueron descartados debido a sus sospechas del procedimiento evaluadas mediante un cuestionario postexperimental.

\section{Procedimiento y diseño}

En cada sesión experimental participaron grupos de 4 ó 5 sujetos que se sentaban frente a una cabina de un aparato de Crutchfield (1955) típico. Cada grupo era asignado aleatoriamente a una de las tres condiciones experimentales. El experimentador explicaba que el aparato se utilizaba para recoger juicios sobre afirmaciones que serían presentadas en diapositivas y leídas en voz alta. El experimentador afirmaba que el propósito del estudio era obtener interpretaciones de enunciados para utilizarlas posteriormente para construir elementos estimulares. Los sujetos recibían un folleto que contenía todos los enunciados. La tarea requería que los sujetos indicaran en una escala el significado e interpretación de una palabra o frase clave subrayada en cada afirmación (los sujetos no podían, por supuesto, ver las respuestas dadas por otros participantes). El experimentador subrayaba que no estaba interesado en las propias opiniones o juicios de los sujetos, sino sólo en su interpretación del significado de la palabra o frase clave de cada elemento.

En las condiciones de presión unánime y apoyo social, el experimentador mencionaba que las respuestas (de acuerdo o en desacuerdo) dadas por un grupo de cuatro personas serían presentadas junto con cada ítem. Este grupo había participado supuestamente en el experimento el día anterior, cuando se recogían juicios en lugar de definiciones. Las respuestas del grupo ficticio se indicaban por señales luminosas en el panel de la cabina de cada sujeto. El mostrar estas respuestas a los sujetos se justificaba como un medio de demostrar el uso típico de los elementos estimuladores que ellos iban a definir.

La manipulación experimental consistía en variar las respuestas evaluativas dadas por el grupo ficticio de cuatro personas de la siguiente forma:

1. Presión unánime. - Los sujetos observaban respuestas que revelaban que 
en los seis elementos críticos de opinión otras personas daban respuestas impopulares. En dos ensayos neutrales, las respuestas del grupo eran contestaciones modales o populares.

2. Apoyo social.-La persona 4 del grupo ficticio daba respuestas populares en todos los ensayos. Las personas 1, 2 y 3 respondían de la misma forma que en la condición de presión unánime.

3. Ausencia de retroalimentación (control).-En esta condición no se decía nada acerca de los juicios de otro grupo, y la presentación de los elementos no iba acompañada de ninguna respuesta de grupo.

\section{ESTIMULOS}

La serie de estímulos consistía en ocho elementos de opinión que trataban de actitudes hacia una variedad de temas. En cada elemento, las respuestas de los supuestos miembros de otro grupo eran presentadas en una escala de nueve puntos ordenados desde «desacuerdo muy fuerte» hasta «acuerdo muy fuerte».

\section{TABLA 1}

Cambio en el significado de los estímulos en función del contexto social (experimento 1)

\begin{tabular}{lcc}
\hline \multicolumn{1}{c}{ Condición } & n & Interpretación \\
\hline Presión unánime & 32 & $30.41 \mathrm{a}$ \\
Apoyo social & 30 & $24.23 \mathrm{~b}$ \\
Ausencia de retroalimentación (control) & 27 & $24.85 \mathrm{~b}$ \\
\hline
\end{tabular}

Nota. Las medias mayores indican una respuesta más infrecuente. Las medias con subíndices diferentes difieren al nivel de significación del .01 (prueba de Newman-Keuls).

En las condiciones de presión unánime y apoyo social, se presentaron respuestas muy impopulares en seis de los ocho elementos (un punto de la escala más allá del percentil 95 de la distribución típica). En los dos elementos restantes, los miembros del grupo daban supuestamente respuestas populares (modales). Las alternativas de respuestas populares ocurrían con la misma frecuencia como números altos o bajos de la escala.

En el folleto de respuestas de los sujetos estaban impresas dos definiciones al- ternativas de una palabra o frase clave subrayada que aparecía en cada afirmación de una opinión. Estas definiciones alternativas eran los extremos de una escala de 10 puntos. Una definición era una interpretación corriente de la afirmación de una opinión, y la otra era una interpretación más extensa o infrecuente, según se determinó a partir de los datos del estudio piloto. Abajo se muestra un ejemplo del formato del folleto de los sujetos:

Nunca dejaría mi camino para ayudar a otra persona si ello significara renunciar a algún placer personal.

Dejar mi camino en la frase significa:

Molestarme

5678910
1234

Arriesgar mi vida 


\section{METODO Y ANALISIS}

Las respuestas de los sujetos en cada ítem se transformaron de manera que las puntuaciones mayores representaban la interpretación más extrema o infrecuente de la palabra o frase subrayada. Se derivó una única puntuación para cada sujeto sumando las respuestas de los seis ítems críticos. Los datos se analizaron según un diseño ínter-sujetos, con las condiciones experimentales y el sexo de los sujetos como factores. Aunque en cada sesión participaran varios sujetos, sus datos eran independientes, puesto que no ocurrió ninguna interacción entre ellos.

\section{RESULTADOS Y DISCUSION}

Se realizó un análisis de varianza de los datos que representaban la interpretación hecha por los sujetos del significado de las palabras o frases clave de los seis elementos críticos. Los resultados del análisis de 3 (condiciones) $\times 2$ (sexo) revelaron un efecto significativo para las condiciones, $F(2,83)=7,58$, $\mathrm{p}<.01$; no hubo ninguna interacción con el sexo de los sujetos. El examen de las medias de las tres condiciones presentadas en la tabla 1 revela que la puntuación más alta se encontró en la condición de presión unánime, apareciendo puntuaciones más bajas en las condiciones de apoyo social y ausencia de retroalimentación.

La dirección de la diferencia entre los tres tipos de condiciones es especialmente notable. Los sujetos de la condición de presión unánime dieron más interpretaciones extremas o infrecuentes a las frases clave de las manifestaciones (puntuaciones más altas) que los sujetos de las otras condiciones. Las comparaciones por pares entre las medias fueron realizadas con una prueba de NewmanKeuls (Winer, 1971). La condición de presión unánime difería significativamente tanto de la condición de apoyo social $(\mathrm{p}<.01)$ como de la condición de ausen- cia de retroalimentación $(\mathrm{p}<.01)$. Las medias de las condiciones de apoyo social y ausencia de retroalimentación no diferían de forma significativa.

Los resultados indicaban que tenía lugar una reestructuración cognitiva sobre los ítems de opinión cuando se informaba a los sujetos sobre las respuestas impopulares dadas previamente por un grupo unánime. Cuando los sujetos se enfrentaban a respuestas impopulares de un grupo unánime el significado que atribuían a los ítems de opinión sufría un cambio: El significado del elemento se desviaba hacia la interpretación más infrecuente. En la condición de apoyo social, en que una persona del grupo confirmaba la opinión personal del sujeto, la interpretación que hacía el sujeto de la afirmación era idéntica a la interpretación hecha en ausencia de retroalimentación (condición de control).

Estos descubrimientos indican que cuando uno se enfrenta con una posición unánime, el proceso de reestructuración cognitiva - reinterpretación del estímulo- puede contribuir significativamente a la conformidad. Lo que hemos propuesto ha sido que la reestructuración cognitiva facilita la conformidad como sigue: En primer lugar, el grupo unánime provoca un cambio en el significado del estímulo, y en segundo, el significado revisado de la afirmación hace la postura del grupo más aceptable, lo cual da como resultado un acercamiento al grupo. Los datos del experimento 1 apoyan la primera parte de la secuencia que proponemos -que las respuestas unánimes de un grupo provocan una desviación en el significado del estímulo. Pero el segundo vínculo supuesto -que la desviación del significado lleva a un mayor acuerdo con la postura del grupo- no se ha sometido a comprobación empírica.

Antes de citar un estudio que prueba el supuesto vínculo entre un cambio en el significado y un cambio de opinión, debemos considerar una explicación alternativa plausible de estos resultados. Es posible que los sujetos pudieran simplemente haber estado informando sobre 
su estimación de la interpretación que hace el grupo del significado de cada estímulo más que dando su propia interpretación. Puesto que esta explicación no puede quedar excluida por los datos disponibles, se diseñó otro experimento como réplica y extensión del experimento 1 .

\section{EXPERIMENTO 2}

En este experimento, los sujetos no sólo daban sus propias respuestas a cada afirmación (como en el experimento 1), sino que también informaban sobre su estimación de la interpretación dada, bien por el grupo o bien por el aliado del sujeto. Se hicieron tres predicciones específicas: a) La propia definición de los sujetos sobre los elementos estimulares debería reproducir los resultados encontrados en el experimento 1; es decir, las definiciones dadas en la condición del grupo unánime deberían ser menos comunes que en las condiciones de apoyo social. b) Cuando se pide que predigan como definen los ítems los miembros del grupo, los sujetos deberían informar que los miembros de la mayoría oponente (tanto en la condición de unanimidad como en la de apoyo social) definen los ítems de forma diferente al aliado del sujeto. c) Finalmente, la propia interpretación del sujeto deberá ser similar a la interpretación que percibe como mantenida por el aliado del sujeto en las condiciones de apoyo social y por la mayoría en la condición de unanimidad.

\section{METODO}

\section{Diseño y procedimiento}

En general, el procedimiento era idéntico al del Experimento 1, con sólo unas pocas modificaciones. Como en el Experimento 1 , se decía a los sujetos que el estudio estaba relacionado con el significado de palabras y que ellos examinarían las respuestas a varios ítems de ac- titud que habían dado una o más personas distintas. La tarea de los sujetos era indicar cómo definía(n) la(s) otra(s) persona(s) ciertas palabras o frases claves en cada enunciado de actitud. Los sujetos recibieron el mismo conjunto de ítems utilizado en el Experimento 1, que incluía las supuestas respuestas de una o más personas. Las presuntas respuestas eran las mismas que las utilizadas en el Experimento 1, pero se empleó un método diferente para presentarlas. En vez de usar un aparato de Crutchfield, las falsas respuestas se colocaron en el cuestionario de cada sujeto. El cuestionario también contenía escalas que servían como medidas dependientes. Utilizando las mismas escalas que en el Experimento 1, los sujetos estimaban cómo definían las palabras subrayadas de cada frase los miembros del grupo o el aliado del sujeto. Después de completar los ocho ítems estimulares, se daba a los sujetos el mismo cuestionario otra vez, con instrucciones de dar su propia interpretación de las partes subrayadas de cada afirmación.

Se utilizó un diseño factorial mixto de $3 \times 2$. El factor ínter-sujetos fue las condiciones experimentales (interpretación del grupo unánime, interpretación de grupo con apoyo social, e interpretación de aliado con apoyo social); el factor intra-sujetos era el objetivo de valoración (uno mismo frente a grupo). Las tres condiciones experimentales se describen abajo:

Interpretación de grupo unánime. En esta condición, el grupo daba las mismas respuestas que a la condición de unanimidad del experimento 1 . Después de estimar cómo definía el grupo cada afirmación, los sujetos daban también su propia definición de la parte crítica de cada elemento.

Interpretación del grupo con apoyo social. Esta condición era idéntica a la de interpretación del grupo unánime, con la excepción de que las respuestas de los miembros del grupo no eran unánimes. Como en la condición de apoyo social del experimento 1, un miembro del grupo 
(persona 4) proporciona apoyo social en todos los ítems. Se pedía a los sujetos que indicaran cómo definía la mayoría (personas 1, 2 y 3) la parte subrayada de cada afirmación. Luego, los sujetos daban su propia interpretación personal de los mismos ítems. (En esta condición no se pedía a los sujetos que predijeran también cómo interpretaba su aliado cada afirmación. Se pensó que pedir a los sujetos predicciones tanto del grupo como del aliado podía contribuir a la tendencia a aumentar las diferencias entre la interpretación del grupo y del aliado del sujeto.)

Interpretación de aliado con apoyo social. En esta condición los sujetos estimaban cómo definía el apoyo social (aliado) la frase subrayada de cada elemento. Por lo demás, esta condición era idéntica a la precedente.

\section{SUJETOS}

Los sujetos fueron 36 mujeres, no graduadas, que fueron asignadas aleatoriamente a una de las tres condiciones experimentales.

\section{RESULTADOS Y DISCUSION}

Las respuestas fueron puntuadas de la misma forma que en el experimento 1 . Un análisis de varianza realizado sobre los datos demostró efectos significativos para las condiciones, $\mathrm{F}(2,3)=51.43 \mathrm{p}<$ $<.001$; el objetivo de las valoraciones, $\mathrm{F}(1,33)=8.69, \mathrm{p}<.01$; y una interacción condiciones $\times$ valoraciones, $F(2,33)$ $=4.69, \mathrm{p}<.025$. El examen de la interacción revela que los resultados fueron consistentes con las predicciones. Observando, en primer lugar, datos de las autovaloraciones de los sujetos, puede verse que los resultados corroboran lo que se encontró en el experimento 1 . Las medias pertinentes se muestran en la primera columna de la Tabla 2. Una prueba de Newman-Keuls realizada después indicó que los sujetos dieron más interpretaciones extremas de los ítems críticos en la condición de interpretación de grupo unánime que en las condiciones de interpretación de grupo de apoyo social y aliado con apoyo social ( $\mathrm{ps}<.10$ y $<.05$, respectivamente) (1). Las de condiciones de apoyo social no diferían significativamente.

TABLA 2

Estimación del significado de las afirmaciones (Experimento 2)

\begin{tabular}{lcc}
\hline \multicolumn{1}{c}{ Condición } & \multicolumn{2}{c}{ Objetivo de la estimación } \\
\cline { 2 - 4 } & Uno mismo & $\begin{array}{c}\text { Miembro(s) } \\
\text { del grupo }\end{array}$ \\
\hline Interpretación de grupo unánime & $34.74 \mathrm{a}, \mathrm{b}$ & $38.25 \mathrm{a}$ \\
Interpretación de grupo con apoyo social & $27.67 \mathrm{~b}, \mathrm{c}$ & $39.50 \mathrm{a}$ \\
Interpretación de aliado con apoyo social & $26.17 \mathrm{c}$ & $25.67 \mathrm{c}$ \\
\hline
\end{tabular}

Nota. Los números más altos indican significado más infrecuente. Las medias con diferentes subíndices difieren al nivel de significación de .05 (prueba de Newman-Keuls). $n=12$ en cada una de las tres condiciones.

(1) La diferencia entre las medias de las condiciones de interpretación de grupo unánime e interpretación de grupo con apoyo social, eran más altamente significativas utilizando una comparación más liberal, $t(22)=2.44, \mathrm{p}<.05$. 
Más importantes para este estudio son los datos de percepción de la interpretación de los miembros del grupo, que se muestran en la segunda columna de la Tabla 2. Los resultados del análisis de Newman-Keuls indicaron que los sujetos percibían que en la condición de interpretación de grupo unánime y de interpretación de grupo con apoyo social, el grupo (o mayoría) mantenía interpretaciones más extremas que el aliado del sujeto en la condición de apoyo social con aliado ( $\mathrm{ps}<.05$ para ambos). Las condiciones de interpretación de grupo unánime y de interpretación de grupo con apoyo social no diferían una de la otra. Así pues, cuando la mayoría del grupo daba respuestas infrecuentes o impopulares a los ítems de opinión, los sujetos creían que la interpretación de los miembros del grupo diferían de la interpretación del aliado del sujeto.

Finalmente, se hicieron comparaciones entre las valoraciones que los sujetos dieron para sí mismos y sus estimaciones de cómo definían otros las afirmaciones. Como puede verse en la Tabla 2, no hubo diferencias entre las autovaloraciones de los sujetos y su estimación de la interpretación del grupo en la condición de interpretación de grupo unánime o en la de interpretación de aliado con apoyo social. Pero su estimación de la interpretación del grupo fue más extrema que las valoraciones para sí mismos en la condición de interpretación de grupo con apoyo social $(\mathrm{p}<.05)$. Así pues, los sujetos percibían que la mayoría sostenía interpretaciones infrecuentes de las afirmaciones, pero sus propias interpretaciones no se vieron afectadas cuando contaban con un aliado.

En resumen, los resultados indicaron que los descubrimientos del experimento 1 no pueden atribuirse al hecho de que los sujetos hayan respondido simplemente según la norma —es decir, de acuerdo a lo que ellos creían que era el significado de los miembros del grupoen lugar de dar su propia interpretación de los elementos. Si los sujetos estuvieran, en efecto, copiando simplemente las «normas» sostenidas por la mayoría, entonces su propia definición de las afirmaciones en la condición de interpretación de grupo con apoyo social debería haber sido la misma que la interpretación que atribuían a la mayoría del grupo. Los resultados del experimento 2 mostraron claramente que éste no era el caso.

Ahora podemos volver a una consideración del vínculo hipotético entre cambio en el significado del estímulo y conformidad. El experimento 3 fue diseñado para proporcionar una prueba fuerte de la conexión hipotética entre una desviación en el significado del estímulo y el desplazamiento resultante de la opinión hacia la postura del grupo.

Supóngase que el significado de un estímulo es idéntico al significado realmente expresado por los sujetos cuando observaron las respuestas impopulares de un grupo unánime. Según nuestro análisis, las propias opiniones expresadas por los sujetos, deberían «imitar» una respuesta de conformidad, incluso aunque la presión del grupo no esté claramente presente. En otras palabras, se puede crear el significado del estímulo que habría existido como consecuencia del proceso de reestructuración cognitiva. Cuando se expone a los sujetos a tal interpretación del estímulo, nuestra predicción es que ellos desviarán su opinión de forma significativa y en una dirección especificable, a saber, hacia la postura mantenida por el grupo que produjo esa interpretación. Así pues, predecimos un cambio significativo hacia la postura del grupo unánime empleado en el experimento 1 .

\section{EXPERIMENTO 3}

Para probar el círculo hipotético entre un cambio en el significado del estímulo y el desplazamiento hacia la postura del grupo, a los sujetos del experimento 3 se les presentó la puntuación modal que 
reflejaba el significado de cada estímulo, según se había determinado por las respuestas dadas por los sujetos del experimento 1. Se pidió a los sujetos que dieran su propia opinión acerca de cada estímulo en dos condiciones. Para cada ítem los sujetos recibieron una puntuación que indicaba, o bien la interpretación de cada estímulo según había sido hecha por los sujetos del experimento 1 , después de haber observado las opiniones de un grupo unánime, o bien la interpretación de cada estímulo dada por los sujetos de control del experimento 1 (que no estaban expuestos a las opiniones de un grupo).

La hipótesis es que el significado que los sujetos aplican a una información influirá en su propia opinión sobre la afirmación. Una definición más atípica o infrecuente dada al elemento debería dar como resultado una buena disposición por parte de la persona a aceptar una respuesta más infrecuente. Como ejemplo, considérese el siguiente elemento empleado en el experimento 1: «Nunca abandonaré mi camino para ayudar a otra persona si ello significa renunciar a algún placer personal.» Si una persona interpreta «abandonar mi camino» en el sentido de «molestarme», entonces posiblemente estará en desacuerdo con la afirmación. Tanto el significado de la afirmación como la respuesta de la persona son semejantes a la que da la mayoría de la gente. Por el contrario, si «abandonar mi camino» es interpretado en el sentido de «arriesgar mi vida», entonces una persona puede muy bien estar de acuerdo con la afirmación. Así pues, nuestra hipótesis es que existe una correspondencia entre el significado de un estímulo y la actitud de uno hacia él. Cuando el significado de una afirmación de opinión se desvía hacia una postura más inusual o infrecuente como resultado de la observación de las respuestas unánimes de un grupo, el sujeto debería estar más dispuesto a aprobar la opinión que cuando la afirmación se define de una forma más típica.

\section{METODO}

\section{Sujetos}

Los sujetos eran 34 no graduados (17 varones y 17 mujeres) de una gran universidad estatal. Catorce sujetos eran miembros de una clase de psicología y se ofrecieron voluntarios al experimento; los demás fueron pagados por participar.

\section{Procedimiento y diseño}

Los sujetos recibieron un cuestionario que contenía una afirmación de opinión y las medidas dependientes pertinentes en cada página. Las instrucciones afirmaban que el cuestionario era parte de un estudio referente a la clasificación de actitudes y el significado de palabras. Las instrucciones indicaban que se incluiría cierta información sobre los significados recogidos anteriormente de miembros de una clase de psicología. La siguiente definición o interpretación de cada palabra o frase era presentada debajo de la afirmación. Se recalcó que esta información era la interpretación de la afirmación dada por personas de otra clase, no su opinión, y que la tarea del sujeto era dar su propia opinión sobre la afirmación.

Las ocho afirmaciones de opinión eran los ítems empleados en los experimentos 1 y 2. Una palabra o frase clave de cada afirmación estaba subrayada, y la supuesta interpretación de otra clase se daba debajo de cada elemento presentando un número rodeado con un círculo en una escala de 10 puntos. Los extremos de la escala eran fijados con una interpretación frecuente y una infrecuente de la palabra o frase clave.

En la mitad inferior de cada página se presentaba de nuevo la afirmación de opinión, y los sujetos indicaban su acuerdo o desacuerdo rodeando un número en una escala de 9 puntos, ordenada de 1 (desacuerdo muy fuetre) a 9 (acuerdo muy fuerte).

Los sujetos fueron asignados aleato- 
riamente a una de las dos condiciones siguientes:

1. Interpretación producida por consenso. La clase dio supuestamente interpretaciones inusuales a seis de los ocho elementos de opinión. La alternativa rodeada con un círculo en la escala de 10 puntos correspondía a la respuesta modal dada por sujetos de la condición de presión unánime del experimento 1. Por ejemplo, considérese el siguiente elemento: «Yo nunca abandonaré mi camino para ayudar a otra persona si ello significase renunciar a algún placer personal». La expresión «abandonaré mi camino» podría ser interpretada en el sentido de «molestarme» (punto 1 de la escala), o, de forma más extremada, en el sentido de «arriesgar mi vida» (punto 10 de la escala), o como intermedia entre estos puntos. En el ejemplo citado, la respuesta modal de cuatro sujetos de la condición de presión unánime del experimento 1 era cinco. Por lo tanto, el punto 1 de la escala aparecía rodeado con un círculo en los folletos de los sujetos de la condición de interpretación típica.

\section{METODO DE ANALISIS}

Las respuestas de cada sujeto a los seis ítems críticos de opinión fueron transformadas de forma que las puntuaciones mayores indicaban una respuesta en la dirección de las contestaciones dadas por el grupo unánime del experimento 1 , es decir, hacia la opinión más atípica $o$ infrecuente. Las puntuaciones transformadas se sumaron para proporcionar una puntuación única para cada sujeto. Los datos fueron analizados según un diseño de $2 \times 2$, con las condiciones experimentales y el sexo del sujeto como factores.

\section{RESULTADOS Y DISCUSION}

El análisis de varianza sobre los datos de actitud reveló un efecto significativo de las condiciones experimentales, $F(1,30)=4.76, \quad p<.05$; el efecto del sexo no fue significativo. Según lo predicho, los sujetos de la condición de interpretación producida por consenso aceptaron una opinión más infrecuente o extrema que los sujetos de la condición de interpretación típica (20.47 frente a 16.06 , respectivamente). Así pues, la exposición a diferentes interpretaciones de una afirmación dio como resultado el que los sujetos expresaran actitudes divergentes hacia ella. Es importante señalar la naturaleza de la diferencia en cuanto a la actitud expresada en función del significado del elemento. En comparación con la actitud de los sujetos que observaron la interpretación típica de los elementos, los sujetos a los que se informó del significado producido por el grupo consensual del experimento 1 expresaron actitudes que estaban más cerca de la postura de ese grupo. Es decir, los datos proporcionan un apoyo para el hipotético vínculo entre un cambio en el significado producido por consenso y el desplazamiento de la actitud de los sujetos para acercarse a la postura del grupo, lo cual es la definición usual del término conformidad.

En resumen, se diseñaron tres experimentos para obtener datos empíricos relativos a la explicación de la conformidad basada en la reestructuración cognitiva. La teoría supone un proceso de dos pasos: en primer lugar, cuando la opinión de uno difiere de la de un grupo unánime, se redefine o se reinterpreta el objeto estimular; $y$ en segundo lugar, la reestructuración del significado del estímulo lleva, a su vez, a un cambio en la respuesta propia. Así pues, para utilizar la terminología de Asch (1948), el pro ceso de influencia del grupo es mediado por un cambio en el objeto de juicio (significado del estímulo) más que por un cambio en el juicio sobre un objeto constante. Los resultados del experimento 1 mostraron que las opiniones de un grupo consensual pueden influir en la interpretación que una persona hace de un objeto (manifestación de opinión). El experimento 2 demostró que el significado percibido no era debido a la simple 
adhesión de los sujetos a la supuesta interpretación de la mayoría del grupo. El experimento 3 mostró que una diferencia en el significado percibido de un objeto estimular afectará el juicio de un individuo (acuerdo-desacuerdo) sobre ese objeto.

En conjunto, los resultados de los tres experimentos proporcionan un fuerte apoyo a la hipótesis de que la reestructuración cognitiva del estímulo es uno de los mecanismos que determina el grado de desplazamiento de una persona hacia la postura de un grupo unánime (es decir, conformidad). Además, la reestructuración cognitiva parecería ser un factor importante que atribuye a la reducción dramática del conformismo producida por el apoyo social (un aliado). Según nuestros análisis, la presencia de un aliado en el grupo evita la ocurrencia de un cambio en el significado del estímulo que si no tendría lugar cuando un individuo se enfrenta a un grupo unánime. Quizá el acuerdo del aliado afirma la creencia del sujeto en lo apropiado de su interpretación del estímulo. Consistentes con este análisis son los datos del experimento 1 que indican que se produjeron significados del estímulo casi idénticos en las condiciones de apoyo social y control (ausencia de retroalimentación).

No afirmamos que la reestructuración cognitiva pueda proporcionar una explicación completa de la conformidad en todas las situaciones. Nuestro análisis parece que sería más directamente aplicable a estímulos subjetivos (por ejemplo, elementos de opinión) que a estímulos objetivos (por ejemplo, juicio de la longitud de líneas). $\mathrm{Ni}$ siquiera por eso debería excluirse por completo la reestructuración cognitiva en el caso de estímulos subjetivos. Algunos de los sujetos de Asch (1956) mantenían que ocurría realmente un cambio perceptual en los estímulos cuando el grupo daba unánimemente respuestas incorrectas sobre juicios de líneas. Quizá los informes de estos sujetos podrán interpretarse más sencillamente como un intento de racionalizar su conducta conformista; no obs- tante es posible que un grupo consensual pudiera tener un impacto directo en la percepción de estímulos objetivos o en el significado de las instrucciones asociadas con los estímulos.

En un trabajo piloto para estos estudios, presentamos a los sujetos una serie de juicios de longitud de líneas. Después de que vieran respuestas de presión procedentes de otros (como se describe en el experimento 1), les pedíamos que eligieran, entre varios dibujos de la configuración estimular, cuál se parecía más a la configuración que acababa de ver. La prueba de reestructuración cognitiva, comparable a la encontrada con los elementos de opinión, consistiría en que los sujetos eligieran modelos que hicieran más correctas las respuestas de la mayoría (es decir, redujeran la discrepancia entre la longitud de la línea elegida por la mayoría y la línea de comparación). Sólo unos pocos sujetos mostraron alguna indicación de reestructuración cognitiva de los elementos cognitivos. Este hallazgo sirve para recordarnos que la reinterpretación cognitiva está limitada por la ambigüedad percibida del elemento. En la medida en que la ambigüedad es baja, la latencia de la reinterpretación disminuye.

$\mathrm{La}$ investigación pasada relacionada con el conformismo y el inconformismo ha tendido a subrayar variables motivacionales y de información más que procesos cognitivos más amplios. El análisis de la reestructuración cognitiva dirige nuestra atención hacia un factor importante que ha sido pasado por alto en explicaciones anteriores de la conformidad y el apoyo social: el impacto del consenso del grupo es el significado percibido del estímulo mismo. El análisis de reestructuración resume el aforismo de la Gestalt de que las propiedades de una entidad (por ejemplo, el significado de un estímulo) está determinada por su relación con otras entidades de la situación (por ejemplo, las interpretaciones que se cree que mantienen los otros). Es conveniente volver a este tema después de que ha estado latente durante tanto 
tiempo (Asch, 1948). Entre tanto, los investigadores han buscado factores externos, situacionales, que afectan a la influencia social (por ejemplo, Allen, $1965,1975)$. La popularización de las teorías de la atribución, sin embargo, ha suscitado un renovado interés por la cuestión de cómo interpreta el individuo los hechos sociales antes de responder a ellos. (Ver Ross, Bierbraner y Hoffman, 1976, para una aplicación de la teoría de la atribución al conformismo). El análisis de reestructuración cognitiva de la conformidad puede ser considerado en términos de atribución como un intento por parte de las personas de encontrar una causa plausible de la conducta aparentemente ilógica de la mayoría. Según se expone aquí, la conducta de la mayoría puede parecer razonable si los que la perciben la atribuyen a diferencias en las definiciones de los elementos estimulares.

Es tentador generalizar a partir de nuestros experimentos sobre conformidad a otros terrenos de influencia social que puedan ser interpretados mediante la hipótesis de reestructuración. Por ejemplo, se ha utilizado una explicación de reestructuración para ayudar a explicar hallazgos en la literatura de intervención del espectador (Latane y Darley, 1969). La vacilación por parte de otros puede cambiar la definición que hace el sujeto de una crisis por una más benigna. Para citar otro ejemplo, los estudios de obediencia (Milgram, 1963) pueden interpretarse de una manera consistente con la hipótesis de la reestructuración. $\mathrm{La}$ presencia de un experimentador de alto prestigio puede provocar que los sujetos noveles definan la situación como menos peligrosa para la víctima de lo que le parecería al observador informado. Una esplicación similà podtía dar cuenta de muchos casos de cambio de actitud inducidos por alta credibilidad del comunicador (por ejemplo, McGuire, 1969). En resumen, sospechamos que una interpretación de reestructuración cognitiva puede aplicarse genéricamente a muchas situaciones de influencia social más allá del paradigma de conformidad examinado en nuestros estudios.

\section{Referencias bibliográficas}

Allen, V. L.: Situational factors in conformity. En L. Berkowitz (Ed.), Advances in experimental social psychology (Vol. 2). Nueva York: Academic Press, 1965.

Allen, V. L.: Social support for nonconformity. En L. Berkowitz (Ed.), Advances in experimental social psychology (Vol. 8). Nueva York: Academic Press, 1975.

Asch, S. E.: Studies in the principles of judgments and attitudes: II. Determination of judgments by group and by ego standards. Journal of Social Psychology, 1940, 12, 433-465.

Asch, S. E.: The doctrine of suggestion, prestige, and imitation in social psychology. Psychological Review, 1948, 55, 250-276.

Asch, S. E.: Effects of group pressure upon the modification and distortion of judgments. En H. Guetzkow (Ed.), Groups, leadersbip and men. Pittsburgh, Pa.: Carnegie Press, 1951.

Asch, S. E.: Studies of independence and submission to group pressure: I. A minority of one against a unanimous majority. Psychological Monographs, 1956, 70 (9, Whole No. 417).

Crutchfield, R. S.: Conformity and character. American Psychologist, 1955, 10, $191-198$.

LatanÉ, B., y Darley, J. M.: Bystander «apathy». American Scientist, 1969, 54, $244-268$.

McGulre, W. J.: The nature of attitudes and attitude change. En G. Lindzey \& E. Aronson (Eds.), Handbook of social psycbology (Vol. 3). Reading, Mass.: Addison-Wesley, 1969.

Milgram, S.: Behavioral study of obedience. Journal of Abnormal and Social Psycbology, 1963, $67,371-378$.

Newtson, D., y Czerlinsky, T.: Adjustment of attitude communications for contrasts by extreme audience. Journal of Personality and Social Psycbology, 1974, 30, 829-837.

Ross, L.; Bierbrauer, G., y Hoffman, S.: The role of attribution processes in conformity and dissent: Revisiting the Asch situation. American Psychologist, 1976, 31, 148-157.

WINER, B. J.: Statistical principles in experimental design (2nd ed.). Nueva York: McGraw Hill, 1971. 